\title{
Identifying cis-mediators for trans-eQTLs across many human tissues using genomic mediation analysis
}

\author{
Fan Yang, ${ }^{1,5}$ jiebiao Wang, ${ }^{1}$ The GTEx Consortium, ${ }^{1,4}$ Brandon L. Pierce, ${ }^{1,2,3}$ \\ and Lin S. Chen ${ }^{1}$ \\ ${ }^{1}$ Department of Public Health Sciences, ${ }^{2}$ Department of Human Genetics, The University of Chicago, Chicago, Illinois 60637, USA; \\ ${ }^{3}$ Comprehensive Cancer Center, The University of Chicago, Chicago, Illinois 60637, USA
}

\begin{abstract}
The impact of inherited genetic variation on gene expression in humans is well-established. The majority of known expression quantitative trait loci (eQTLs) impact expression of local genes (cis-eQTLs). More research is needed to identify effects of genetic variation on distant genes (trans-eQTLs) and understand their biological mechanisms. One common trans-eQTLs mechanism is "mediation" by a local (cis) transcript. Thus, mediation analysis can be applied to genome-wide SNP and expression data in order to identify transcripts that are "cis-mediators" of trans-eQTLs, including those "cis-hubs" involved in regulation of many trans-genes. Identifying such mediators helps us understand regulatory networks and suggests biological mechanisms underlying trans-eQTLs, both of which are relevant for understanding susceptibility to complex diseases. The multitissue expression data from the Genotype-Tissue Expression (GTEx) program provides a unique opportunity to study cis-mediation across human tissue types. However, the presence of complex hidden confounding effects in biological systems can make mediation analyses challenging and prone to confounding bias, particularly when conducted among diverse samples. To address this problem, we propose a new method: Genomic Mediation analysis with Adaptive Confounding adjustment (GMAC). It enables the search of a very large pool of variables, and adaptively selects potential confounding variables for each mediation test. Analyses of simulated data and GTEx data demonstrate that the adaptive selection of confounders by GMAC improves the power and precision of mediation analysis. Application of GMAC to GTEx data provides new insights into the observed patterns of cis-hubs and trans-eQTL regulation across tissue types.
\end{abstract}

[Supplemental material is available for this article.]

Recent studies have demonstrated that many expression quantitative trait loci (eQTLs) that affect expression of local transcripts (ciseQTLs) also affect the expression of distant genes (trans-eQTLs) (Battle et al. 2014; Pierce et al. 2014). This observation suggests the effects of trans-eQTLs are "mediated" by the local (cis-) gene transcripts near the eQTLs (Fehrmann et al. 2011; Pierce et al. 2014). In other words, some cis-eQTLs are also trans-eQTLs because the variation in the expression of the cis-gene affects the expression of a trans-gene or genes. In the simplest scenario, a cis-eQTL affects expression of a nearby gene that is a transcription factor, which then regulates the transcription of a distant gene; thus, the transcription factor "mediates" the effect of the eQTL on the distant gene. By studying eQTLs that have both the cis- and trans-effects, one may identify the cis-genes that mediate the effects of transeQTLs on expression of distant genes, including "cis-hub" genes that regulate the expression of many trans-genes (Chen et al. 2007; Stranger et al. 2012). Studying mediation (causation) moves beyond the analysis of cis- and trans- associations (correlation). Prior studies have applied mediation tests to genome-wide SNPs and expression data (from blood cells) to identify transcripts that

\footnotetext{
${ }^{4}$ A full list of Consortium members and their affiliations is available at the end of the text.

${ }^{5}$ Present address: Department of Biostatistics and Informatics, Colorado School of Public Health, University of Colorado Denver, Aurora, CO 80045, USA

Corresponding authors: Ichen@health.bsd.uchicago.edu,

brandonpierce@uchicago.edu

Article published online before print. Article, supplemental material, and publication date are at http://www.genome.org/cgi/doi/10.1101/gr.216754.116.
}

are cis-mediators of the effects of trans-eQTLs (Chen et al. 2007; Battle et al. 2014; Pierce et al. 2014). Characterizing these regulatory relationships will allow us to better understand regulatory networks and their roles in complex diseases (Veyrieras et al. 2008), as it is well known that SNPs influencing human traits tend to be eQTLs (Nicolae et al. 2010). Analyses of cis-mediation will also provide us with a better understanding of the biological mechanisms underlying trans-eQTLs (Westra et al. 2013).

The expression levels of a given gene can vary substantially across human cell types, and the regulatory relationships between SNPs and gene expression levels may also depend on cell type (Torres et al. 2014; Wang et al. 2016). To date, most large-scale eQTL studies have been conducted using RNA extracted from peripheral blood cells, which are mixtures of different cell types and may not be informative for gene regulation in other human tissues. In order to study gene expression and regulation in a variety of human tissues, the National Institutes of Health commonfund GTEx (Genotype-Tissue Expression) project has collected expression data on 44 tissue types from hundreds of post-mortem donors (Lonsdale et al. 2013; Ardlie et al. 2015). This rich transcriptome data, coupled with data on inherited genetic variation, provides an unprecedented opportunity to study gene expression and regulation patterns from both cross-tissue and tissue-specific perspectives.

() 2017 Yang et al. This article is distributed exclusively by Cold Spring Harbor Laboratory Press for the first six months after the full-issue publication date (see http://genome.cshlp.org/site/misc/terms.xhtml). After six months, it is available under a Creative Commons License (Attribution-NonCommercial 4.0 International), as described at http://creativecommons.org/licenses/by-nc/4.0/. 
One major challenge in mediation analysis is the presence of unmeasured or unknown variables that affect both the mediator (i.e., cis-gene) and outcome (i.e., trans-gene) variables. The presence of such a variable is known as "mediator-outcome confounding," and in such a scenario, estimates obtained from mediation analysis can be biased (Robins and Greenland 1992; Pearl 2001; Cole and Hernan 2002). In other words, in the presence of an unmeasured confounding variable(s), the association between the two cis- and trans-genes will be a biased estimate of the causal relationship between the two genes, and estimates obtained from mediation analysis will be biased. It is well recognized that measures of transcriptional variation can be affected by genetic, environmental, demographic, technical, and biological factors. The presence of unmeasured or unknown confounding effects may induce inflated rates of false detection of mediation relationships or jeopardize the power to detect real mediation, if those confounding effects are not well accounted for. Given that eQTL analyses are conducted in the context of complex biological systems, there is a wide array of biological variables that could potentially confound the mediator-outcome association and bias mediation estimates, a problem that may be exacerbated by the diversity of GTEx participants, with respect to ethnicity, age, and cause of death. Given these challenges, it is desirable to have methods that consider a large pool of potential confounding variables.

To adjust for unmeasured or unknown confounding effects in genomics studies, existing literature focused on the construction of sets of "hidden" variables that capture a substantial amount of the variation in a large set of variables (Price et al. 2006; Leek and Storey 2007; Stegle et al. 2012). A commonality of those approaches is that they model the effects of hidden confounding factors and summarize those effects into a set of constructed variables, sort those variables decreasingly by their estimated impacts, and adjust the top ones as a set of covariates to eliminate major confounding effects in the subsequent analysis. For example, in GTEx eQTL analyses (Ardlie et al. 2015; The GTEx Consortium 2017), the top Probabilistic Estimation of Expression Residuals (PEER) factors were estimated for each tissue type, and up to $35 \mathrm{fac}-$ tors were adjusted. One aspect that is largely ignored is that not all potential gene pairs (or pairs of regulator and regulated genes) are affected by the same set of hidden confounders. There are likely thousands of cis-mediated trans-eQTLs in the human genome, i.e., trios consisting of a genetic variant, a cis-gene transcript, and a trans-gene transcript in a specific tissue type. However, for each trio, mediator-outcome confounding will be present only when a hidden variable is causally related to the regulator and regulated genes. By this criterion, the potential confounder set varies by different trios. Adjusting a universal set of variables for all mediation trios is not only inefficient but also may limit our ability to consider a larger pool of potential confounding variables in genomic mediation analyses.

We propose to adaptively select the variables to adjust for each trio given a large set of constructed or directly measured potential confounding variables. This strategy supplements existing confounding adjustment approaches that focus on the construction of variables for capturing confounding effects and enlarges the pool of variables to be considered. Additionally, by leveraging the cis genetic variant as an "instrumental variable," we are able to select the variables capturing confounding effects rather than variables only correlated with cis- and trans-genes. We further propose a mediation test with nonparametric $P$-value calculation, adjusting for the adaptively selected sets of confounders. We term the proposed algorithm Genomic Mediation analysis with Adaptive
Confounding adjustment (GMAC). The GMAC algorithm improves the efficiency and precision of confounding adjustment and the subsequent genomic mediation analyses. We applied GMAC to each of the 44 tissue types of GTEx data in order to study the trans-regulatory mechanism in human tissues. Our algorithm identifies genes that mediate trans-eQTLs in multiple tissues, as well as "cis-hubs" that mediate the effects of a trans-eQTL on multiple genes.

\section{Results}

\section{GMAC improves power and precision of analysis of GTEx data}

We performed genomic mediation analysis with data from each tissue type in GTEx. Taking the tissue, adipose subcutaneous, as an example, there are 298 samples for this tissue type, and genelevel expression measures for 27,182 unique transcripts are available after quality control. Consider a candidate mediation trio consisting of a gene transcript $i\left(C_{i}\right)$, its cis-associated genetic locus $\left(L_{i}\right)$, and another gene transcript $j\left(T_{j}\right)$ in trans-association with the locus. The goal is to test for mediation of the effect of the genetic locus on the trans-gene by the cis-gene (see Fig. 1). We focused on only the trios $\left(L_{i}, C_{i}, T_{j}\right)$ in the genome showing both cis- and trans-eQTL associations, i.e., $L_{i} \rightarrow C_{i}$ and $L_{i} \rightarrow T_{j}$. Because associations are necessary but not sufficient conditions for inferring mediation, by considering only the trios with cis- and transassociations, we effectively reduced the search space to a promising pool of candidate mediation trios and alleviated the multiple testing burdens. We detected and selected a lead cis-eQTL for 8500 of these transcripts, corresponding to 8216 unique ciseSNPs for subsequent analysis (see Methods). We applied Matrix eQTL (Shabalin 2012) to the 8216 SNPs and the 27,182 gene expression levels to calculate the pair-wise trans-associations. At the $P$-value cutoff of $10^{-5}$, there were 3169 significant pairs of SNP and trans-gene transcripts. Since some cis-eSNPs were the lead cis-eSNPs for multiple local gene transcripts, those significant SNP and trans-gene pairs entailed a total of 3332 trios (i.e., SNPcis-trans) for this tissue type. We applied GMAC (see Methods; see Fig. 2 for a graphical illustration of the main steps of the GMAC) to the 3332 trios in this tissue type to test for mediation

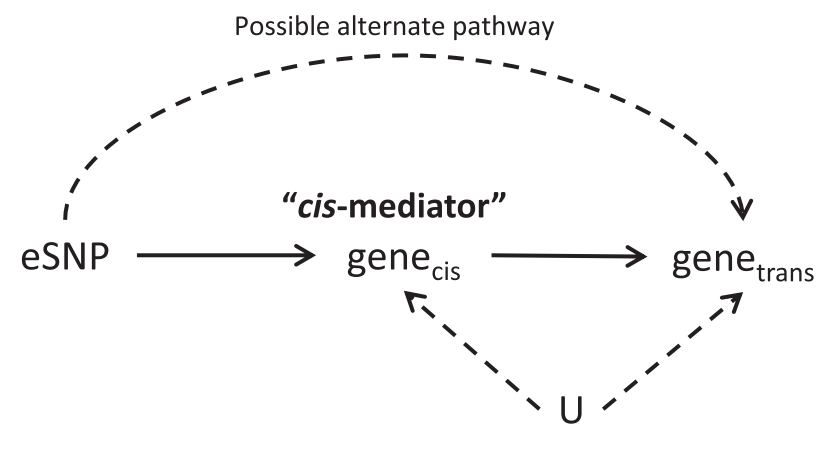

Possible mediator-outcome confounding

Figure 1. Causal diagram demonstrating mediation and "mediator-outcome confounding." Here, the variable set "U" represents a set of unmeasured or unknown variables that may show confounding effects in the mediation analysis. Mediation analysis can detect mediation of the effect of the eSNP on the trans-gene by the cis-gene, assuming mediator-outcome confounding is absent or adjusted for in the analysis. Mediation will not be detected if the effect of the eSNP on the trans-gene is through some alternative pathway that does not involve the cis-gene.

\section{Genome Research}

www.genome.org 


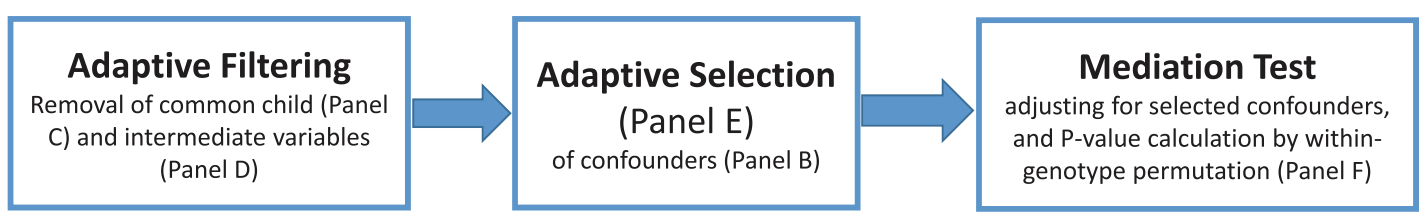

B

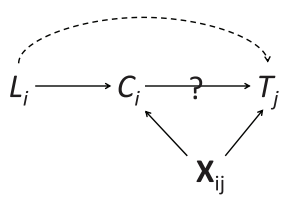

Confounder

$\mathbf{E}$

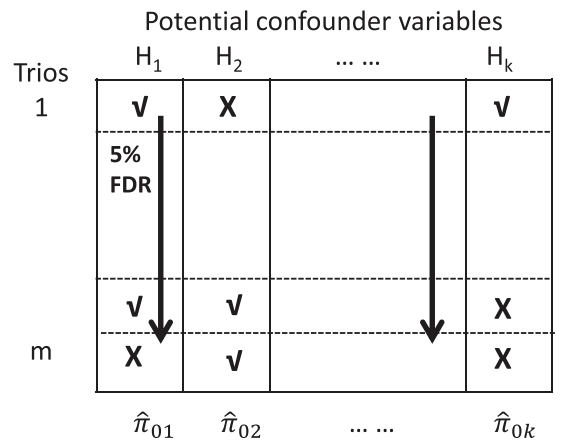

C

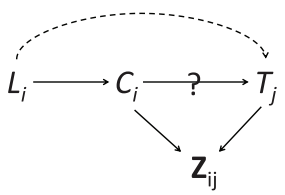

Common Child

F

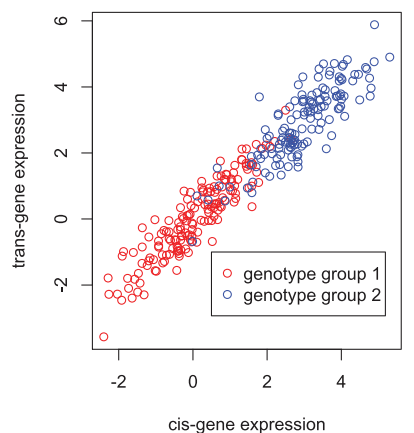

D

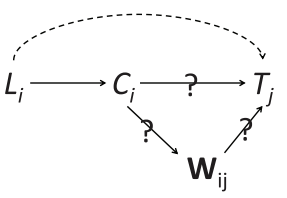

Intermediate Variable

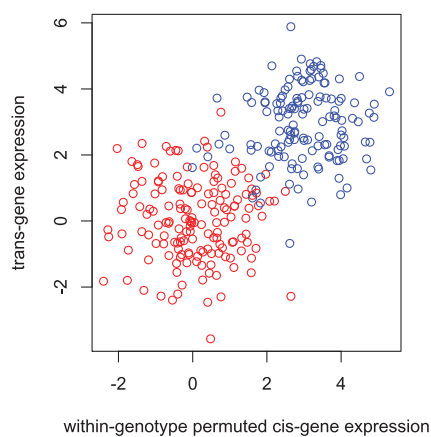

Figure 2. Graphical illustrations of GMAC and its main ideas. $(A) A$ summary of the $G M A C$ algorithm; $(B)$ a mediation relationship among an eQTL, $L_{i}$, its cis-gene transcript, $C_{i}$, and a trans-gene transcript, $T_{j}$, with confounders, $\mathbf{X}_{\mathbf{i j}}$, allowing $L_{i}$ to affect $T_{j}$ via a pathway independent of $C_{i}$ (C) a mediation trio where $C_{i}$ and $T_{j}$ have common child variable(s), $\mathbf{Z}_{\mathbf{i j}} ;(D)$ a mediation trio where $C_{i}$ affects $T_{j}$ through intermediate variable(s), $\mathbf{W}_{\mathbf{i j}}$. (E) The adaptive confounder selection procedure: Based on the $P$-value matrix for the association of each potential confounder variable to at least one of the cis- or the trans-gene transcripts, we apply a stratified FDR approach by considering the $P$-values for each potential confounder (each column) as a stratum, with the significant ones indicated by a check mark $(\sqrt{ })$. When conducting the mediation test for each trio, we only adjust for the significant confounding variables (the ones with $\sqrt{ }$ in each row). (F) A mediation trio $L_{i} \rightarrow C_{i} \rightarrow T_{j}$ (left) and a trio under the null with both cis-linkage and trans-linkage but no mediation (right). Withingenotype permutation of the cis-gene expression levels maintains the cis- and trans-linkage (different mean levels) while breaking the potential correlation between the cis- and trans-expression levels within each genotype group. Note that $\mathbf{X}_{\mathbf{i j}}, \mathbf{Z}_{\mathbf{i j}}, \mathbf{W}_{\mathbf{i j}}$ may vary by trios and are all subsets of $\mathbf{H}$. We assume that either $\mathbf{X}_{\mathbf{i j}}$ or a combination of variables in $\mathbf{X}_{\mathbf{i j}}$ would capture the variation of the unmeasured confounder $\mathbf{U}$ in Figure 1.

and obtained the mediation $P$-values for those trios. Since different tissue types have different sample sizes in GTEx and in addition to cross-tissue confounders, there are many tissue-specific confounding effects, we constructed Principal Components (PCs) from the expression data of each tissue type as potential confounders (Fig. 2B). The number of PCs for each tissue type is equal to the tissue sample size minus 1 . We analyzed trios for mediation in a similar fashion for all other GTEx tissue types.

At the 5\% false discovery rate (FDR) (Storey and Tibshirani 2003) level, we identified 6145 instances of significant mediation out of 64,824 trios tested in the 44 tissue types. These trios represent potential examples of cis-mediation of trans-eQTLs within a specific tissue. Table 1 lists the number of significant mediation trios at 5\% FDR and the number of trios with suggestive mediation ( $P$-value $<0.05)$, as well as the total number of trios with significant cis- and trans-associations for all tissue types. The number of confounders selected for each mediation test ranged from 0 to 22 across all tissue types, with a mean of 7.695 and a median of 8 . The median number of confounders selected for each tissue type ranged from 3 to 12 , while the pool of variables (PCs) from which we selected confounders ranged from 69 to 360 . Supplemental Table S1 presents the descriptive statistics for the number of selected confounders for all the trios in each tissue type. It is clear that with GMAC, on average, we adjust an efficient number while considering a large pool of confounding variables in the mediation tests, and that may improve the power and accuracy of the analyses.

Again taking the tissue, adipose subcutaneous, as an illustration, in Figure 3, we plotted the negative log base 10 of the mediation $P$-values versus the percentages of reduction in transeffects after adjusting for a potential cis-mediator, based on mediation tests without adjusting for hidden confounders (Fig. 3A) and mediation tests by GMAC considering all PCs as potential confounders (Fig. 3B). The percentage of reduction in trans-effects is calculated by $\left(\beta_{2}^{m}-\beta_{2}\right) / \beta_{2}^{m} \times 100 \%$, where $\beta_{2}^{m}$ is the marginal trans-effect of the eQTL on the trans-gene expression levels, and $\beta_{2}$ is the trans-effect after adjusting for cis-mediation. For trios representing true cis-mediation, we expect the trans-effects to be substantially reduced after adjusting for the mediator; that is, we expect the trios with very significant mediation $P$-values 
Table 1. A description of GTEx tissue types and the number of significant instances of mediation (i.e., SNP-cis-trans trios) identified by GMAC

\begin{tabular}{|c|c|c|c|c|}
\hline Tissue name & Tissue sample size & \# Trios tested & $\begin{array}{l}\text { \# Trios with suggestive mediation } \\
\qquad(P \text {-value }<0.05)\end{array}$ & \# Trios significant at 5\% FDR \\
\hline Muscle skeletal & 361 & 2387 & 496 & 264 \\
\hline Whole blood & 338 & 2274 & 508 & 281 \\
\hline Skin sun-exposed lower leg & 302 & 3273 & 629 & 330 \\
\hline Adipose subcutaneous & 298 & 3332 & 640 & 325 \\
\hline Artery tibial & 285 & 2699 & 527 & 281 \\
\hline Lung & 278 & 2762 & 543 & 323 \\
\hline Thyroid & 278 & 3894 & 696 & 376 \\
\hline Cells transformed fibroblasts & 272 & 3000 & 642 & 340 \\
\hline Nerve tibial & 256 & 3812 & 677 & 326 \\
\hline Esophagus mucosa & 241 & 2640 & 465 & 242 \\
\hline Esophagus muscularis & 218 & 2431 & 447 & 230 \\
\hline Artery aorta & 197 & 2009 & 368 & 186 \\
\hline Skin not sun-exposed suprapubic & 196 & 1961 & 365 & 177 \\
\hline Heart left ventricle & 190 & 1290 & 242 & 115 \\
\hline Adipose visceral omentum & 185 & 1410 & 257 & 125 \\
\hline Breast mammary tissue & 183 & 1422 & 254 & 126 \\
\hline Stomach & 170 & 1153 & 235 & 107 \\
\hline Colon transverse & 169 & 1585 & 309 & 161 \\
\hline Heart atrial appendage & 159 & 1221 & 243 & 103 \\
\hline Testis & 157 & 3896 & 607 & 267 \\
\hline Pancreas & 149 & 1270 & 208 & 102 \\
\hline Esophagus gastroesophageal junction & 127 & 857 & 148 & 74 \\
\hline Adrenal gland & 126 & 981 & 185 & 94 \\
\hline Colon sigmoid & 124 & 968 & 220 & 108 \\
\hline Artery coronary & 118 & 874 & 191 & 95 \\
\hline Cells EBV-transformed lymphocytes & 114 & 856 & 152 & 78 \\
\hline Brain cerebellum & 103 & 1295 & 187 & 84 \\
\hline Brain caudate basal ganglia & 100 & 763 & 139 & 61 \\
\hline Liver & 97 & 496 & 87 & 41 \\
\hline Brain cortex & 96 & 754 & 134 & 45 \\
\hline Brain nucleus accumbens basal ganglia & 93 & 592 & 97 & 43 \\
\hline Brain frontal cortex BA9 & 92 & 595 & 102 & 52 \\
\hline Brain cerebellar hemisphere & 89 & 1072 & 222 & 116 \\
\hline Spleen & 89 & 825 & 157 & 68 \\
\hline Pituitary & 87 & 732 & 132 & 61 \\
\hline Prostate & 87 & 474 & 101 & 54 \\
\hline Ovary & 85 & 469 & 95 & 43 \\
\hline Brain putamen basal ganglia & 82 & 481 & 94 & 35 \\
\hline Brain hippocampus & 81 & 343 & 93 & 47 \\
\hline Brain hypothalamus & 81 & 342 & 74 & 41 \\
\hline Vagina & 79 & 248 & 58 & 25 \\
\hline Small intestine terminal ileum & 77 & 434 & 82 & 39 \\
\hline Brain anterior cingulate cortex BA24 & 72 & 365 & 81 & 29 \\
\hline Uterus & 70 & 287 & 62 & 25 \\
\hline
\end{tabular}

to have positive percent reduction in the trans-effect. In Figure $3 \mathrm{~A}$, we observed many trios with significant mediation $P$-values, but for a substantial number of these trios, the percentages of reduction in trans-effects are negative. At the mediation $P$-value threshold of $0.05,1577$ out of 3332 trios were significant; however, 712 trios $(712 / 3332=21.3 \%)$ have negative percent reduction in trans-effects. This contradicting result is expected in the presence of unadjusted confounders, and many of these trios may be false positives. Thus, mediation analyses of GTEx data without adjusting for hidden confounding effects will lead to many spurious findings.

In addition to our main analysis based on GMAC (adaptively selecting confounders from all expression PCs), we also conducted mediation tests adjusting for only the 35 PEER factors used in the GTEx eQTL analyses (The GTEx Consortium 2017). At the 5\% FDR level, 3356 out of 64,824 trios from all tissue types were significant. Using GMAC adjusting for adaptively selected PEER factors, 5131 trios were significant at the 5\% FDR level. The comparison of adjusting for all (up to 35) PEER factors versus GMAC (considering a larger pool of potential confounders with up to 360 PCs) demon- strates that adaptive selection enables more efficient adjustment of confounding effects with much fewer selected confounding variables (Supplemental Table S1) and improves power to detect mediation. Furthermore, using GMAC to adaptively select confounders from all PCs identifies 6145 significant trios, suggesting an increase in power. It can also be seen that all three methods(1) GMAC with adaptively-selected PCs, (2) GMAC with adaptively-selected PEER factors, and (3) adjusting for all PEER factorswould yield reasonable mediation estimates (i.e., percentages of reduction in trans-effects versus mediation $P$-values), compared to no confounder adjustment (see Supplemental Fig. S1). In conclusion, motivated by the fact that the potential confounder set may vary by different trios, GMAC adaptively adjusts for only the variables that are causally related to both cis- and trans-genes and may show confounding effects in the mediation analysis of each trio (Fig. 2B). Compared with adjusting for a universal set of (top) variables for all mediation trios, GMAC considers a larger pool of potential confounding variables in genomic mediation analyses and enjoys improved power while controlling for false positives.

\section{Genome Research}

www.genome.org 
A

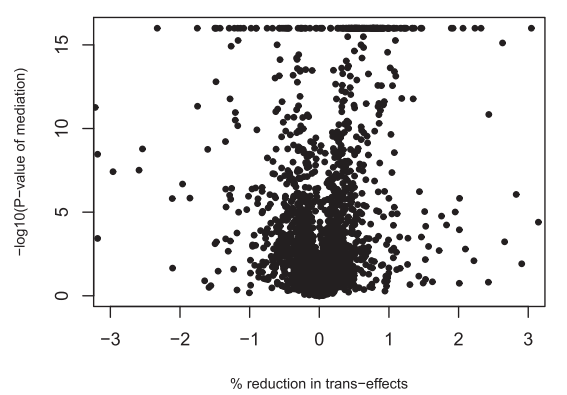

B

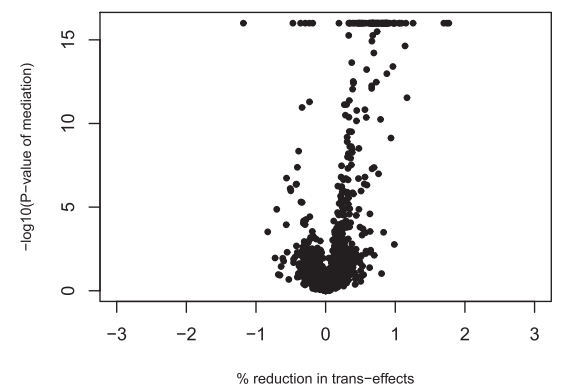

Figure 3. Plots of negative log base 10 of mediation $P$-values versus the percentages of reduction in trans-effects after accounting for cis-mediation. The $P$-values are calculated based on $(A)$ mediation tests without adjusting for hidden confounders, and (B) mediation tests by GMAC considering all PCs as potential confounders. $P$-values are truncated at $10^{-16}$. The plots are based on the results from the adipose subcutaneous tissue. The percentage of reduction in trans-effects is calculated by $\left(\beta_{2}^{m}-\beta_{2}\right) / \beta_{2}^{m} \times 100 \%$, where $\beta_{2}^{m}$ is the marginal trans-effect of the eQTL on the trans-gene expression levels, and $\beta_{2}$ is the transeffect after adjusting for a potential cis-mediator and other covariates. For trios with true cis-mediations, the marginal trans-effects are nonzero, and after adjusting for the true cis-mediators, we expect the adjusted trans-effects $\beta_{2}$ to be substantially reduced; that is, we expect the trios with very significant mediation $P$-values to have positive percent reduction in trans-effects. For results based on no adjustment of hidden confounders $(A)$, we observed many trios with significant mediation $P$-values but the percentages of reduction in trans-effects are negative. At the $0.05 P$-value threshold, $712(21.4 \%)$ and $188(5.6 \%)$ out of 3332 trios have $P$-values below the threshold and percent reduction in trans-effects being negative in $A$ and $B$, respectively.

The majority of the cis-mediators and trans target genes observed among our trios showing mediation have high mappability scores (Supplemental Fig. S2). However, nonuniquely mapping reads can result in false positive eQTLs, so we consider the mappability of each gene as a quality control filter for studying specific examples of cis-mediation (see Methods). Examining the mappability for genes involved in cis-mediation, we observed that cis-genes showing evidence of cis-mediation for multiple trans-genes were enriched for cis-genes with low mappability scores (Supplemental Fig. S2). Similarly, genes showing evidence of cis-mediation across many different tissue types were also enriched for genes showing low mappability scores (Supplemental Fig. S2). This finding demonstrates that transcripts that do not uniquely map to the genome are an important source of false positives when conducting genomic mediation analysis. More specifically, we find that analyzing low-mappability genes can lead to the identification of spurious cis-hubs and cross-tissue cis-mediators.

We attempted to identify "cis-hubs" with high mappability in the GTEx data, defined as a transcript that appears to mediate the effect of a nearby eSNP on expression of multiple distant (i.e., trans) gene transcripts. Restricting our analysis to cis- and transgenes with mappability $>0.95$, we observed 685 cis-genes with at least two trans targets (considering all tissues), representing $21 \%$ of the 3168 cis-genes observed among the trios with a mediation $P$-value $<0.05$ (Table 2 ). In addition, we attempted to identify cis-genes that have at least one trans target in multiple tissues. Restricting to high-mappability genes, we observed 531 cis-genes with trans targets in more than one tissue, representing $17 \%$ of the 3168 cis-genes observed among the trios with a mediation $P$ value $<0.05$ (Table 2). We observed only six examples of cis-genes that had the same trans targets in multiple tissues. In other words, the vast majority of cis-hubs observed were of two distinct types: (1) those that mediated the effect of a trans-eQTL on multiple trans-genes within a single tissue type; and (2) those that were mediators in multiple tissues but with unique trans targets in each tissue type. All instances of cis-mediation of trans-eQTLs with a mediation $P$-value $<0.1(16,648$ trios $)$ are listed in Supplemental Table S2, including trios containing transcripts with low mappability.

\section{Examples of mediation across tissues}

In analyses restricted to cis- and transgenes with mappability scores $>0.95$, one biologically interpretable example of a cis-gene that appears to mediate the effects of trans-eSNPs in multiple tissues is the IFI44L gene on Chromosome 1 (Fig. 4A). IFI44L is a cis-eGene in two GTEx tissues (cerebellar hemisphere and tibial nerve), and the cis-eSNPs associated with IFI44L expression are also associated with expression of multiple genes in trans in both cerebellar and tibial nerve tissue. OAS1 is a trans target of these SNPs in both tissues, while other trans targets are observed in only cerebellar (AGRN and PARP12) or tibial nerve (RSAD2, OAS2, and EPSTI1). Below the mappability threshold of 0.95 , we observe an additional potential trans target of $I F I 44 L$, present in both cerebellar and tibial nerve tissue, IFIT3 (mappability $=0.87$ ). These relationships are depicted in Figure 4A.

Interestingly, if we expand our analysis to include cis- and trans-genes with mappability $>0.90$, we detect IFI44 (mappability of 0.93 ) as a cis-mediator regulating a nearly identical set of trans genes across three tissues: cerebellar hemisphere (OAS1, IFIT2, $A G R N$, and PARP12), tibial nerve (OAS1, IFIT3, RSAD2, and EPSTI1), and sun-exposed skin (IFIT1) (Fig. 4B). IFI44 and IFI44L are paralogs and reside adjacent to each other on $1 \mathrm{p} 31.1$. These genes are regulated by the same SNP in each tissue. It is highly unlikely that sequence similarity between these two genes causes our RNAseq-based expression measurements for IFI44 (and/or IFI44L) to reflect the expression variation of both genes. The two regions of similarity shared between these two transcripts are 753 and 202 bp in length, and these regions share $69 \%$ and $67 \%$ similarity, respectively (Supplemental Fig. S3). These two regions contain no identical sequences longer than $\sim 10 \mathrm{bp}$, making it impossible for an RNA-seq read (76 bp) to be ambiguous in terms of its mapping to IFI44 vs. IFI44L. Furthermore, a prior study of array-based

Table 2. Frequency of cis-genes that mediate the effect of a transeQTL on multiple trans-genes or in multiple tissue types

\begin{tabular}{lcccc}
$\begin{array}{l}\text { Observed number of trans targets } \\
\text { for each cis-gene }\end{array}$ & & \multicolumn{2}{c}{$\begin{array}{c}\text { Number of tissues for which } \\
\text { each cis-gene is a mediator }\end{array}$} \\
\cline { 1 - 2 } \cline { 5 - 6 } $\begin{array}{c}\text { Number of trans } \\
\text { targets }\end{array}$ & $\begin{array}{c}\text { Cis-gene } \\
\text { count }\end{array}$ & & $\begin{array}{c}\text { Number of } \\
\text { tissues }\end{array}$ & $\begin{array}{c}\text { Cis-gene } \\
\text { count }\end{array}$ \\
\hline 1 & 2510 & & 1 & 2637 \\
2 & 482 & & 2 & 420 \\
3 & 123 & & 3 & 91 \\
4 & 33 & & 4 & 16 \\
$5-6$ & 12 & & 5 & 3 \\
$7-14$ & 8 & & 6 & 1
\end{tabular}


A

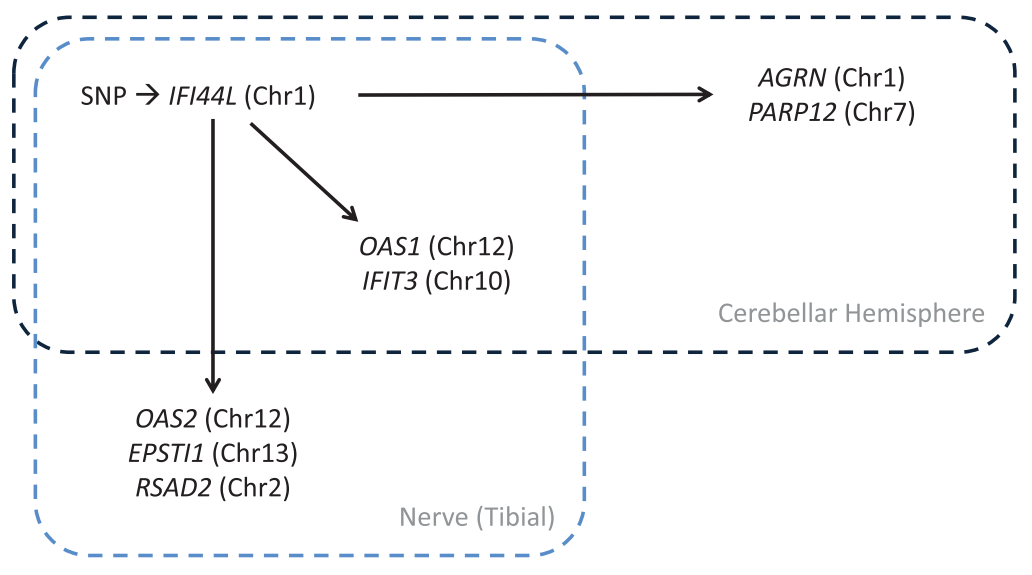

B

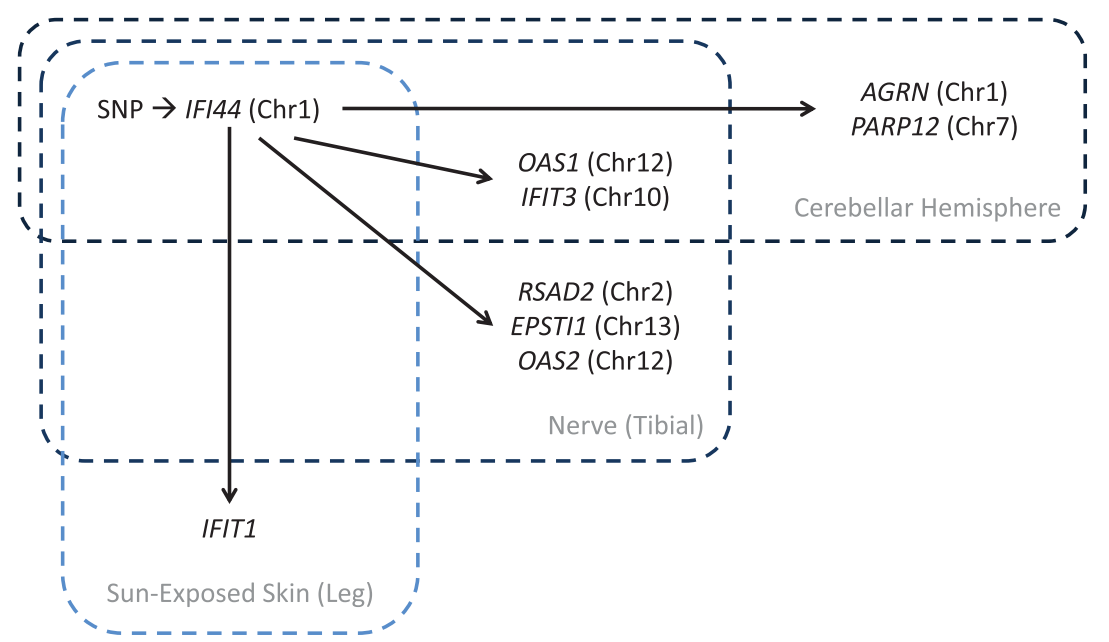

Figure 4. A biologically interpretable example of a cis-eGene (IF/44L) that appears to mediate the effects of trans-eSNPs in multiple tissues. The gene IFI44L $(A)$ resides $<5 \mathrm{~kb}$ away from IFI44 (B), and expression of these genes is associated with a common cis-eQTL that also impacts the expression of multiple genes in trans in multiple tissues. Both IFI44 and IFI44L show statistical evidence of mediation for a similar set of interferon-related genes. Thus, based on this evidence, we infer that at least one of these genes is a cis-mediator, although we cannot know which is (or if both are) the true mediator.

cell lines (Cheon and Stark 2009), virusinfected airway epithelial cell cultures (Ioannidis et al. 2012), and peripheral blood of individuals with acute respiratory infections (Zaas et al. 2009), as well as in both normal and cancerous human tissue (Cancer Cell Metabolism Gene DB, https://bioinfo.uth.edu/ccmGDB/). These previously reported co-expression findings also extend to EPSTI1 (Cheon and Stark 2009), the one gene we find to be a trans target of IFI44L (and/or IFI44) that does not have a well-established function in immune response, providing additional evidence of an immune-related function for this gene.

Variation in the IFI44L gene is associated with risk for MMR (measles, mumps, and rubella) vaccination-related febrile seizures, with a missense variant in IFI44L showing the strongest association (Feenstra et al. 2014). Variation in IFI44L has also been implicated in schizophrenia risk (Ruderfer et al. 2014) as well as bipolar disorder (Chen et al. 2013). These findings suggest that the putative cross-tissue cis-hub identified here may be relevant to multiple neurological and psychological disorders, particularly those with etiologies related to immune function.

\section{Comparison of GMAC with other methods using simulated data}

We evaluate the performance of the proposed GMAC in various simulated data scenarios. For each scenario described below, we simulated 1000 mediation trios $\left(L_{i}, C_{i}, T_{j}\right)$ for a sample size of $n=350$, similar to the sample size of the GTEx data. Each mediation trio consists of a gene transcript $i\left(C_{i}\right)$, its cis-associated genetic locus $\left(L_{i}\right)$, and a gene transcript $j$

expression measures in endometrial cancer tissue reported genetic coregulation of IFI44 and IFI44L (in trans) (Kompass and Witte 2011).

Based on these observations, it is unclear which of these two genes is truly a cis-mediator of the observed trans-eQTLs (or if both are mediators). The causal cis-eSNP for IFI44 (and/or IFI44L) appears to be different in different tissues, as the LD between the lead cis-eSNPs in cerebellar (rs12129932) and the lead eSNP in tibial nerve (rs74998911) is quite low, with $r^{2}<0.01$ in EUR 1000 Genomes data (The 1000 Genomes Project Consortium 2015).

Regardless of the uncertainty whether IFI44L or IFI44 (or both) is the true cis-hub of this trans-eQTL, nearly all of the genes involved in the putative regulatory pathways identified here are interferon-regulated/inducible genes, namely OAS1, OAS2, IFIT1, IFIT3, IFI44, IFI44L, RSAD2, and AGRN (Cheon and Stark 2009; Kyogoku et al. 2013). These genes have been previously reported to be co-expressed and/or coregulated in various human cell types, including interferon-exposed fibroblasts and mammary epithelial
$\left(T_{j}\right)$ in trans-association with the locus. Note that, in the mediation analysis in this work (simulations and real data analysis), we consider only the trios with evidence of cis- and trans-associations, $L_{i} \rightarrow C_{i}$ and $L_{i} \rightarrow T_{j}$. We are interested in testing whether an observed trans-eQTL association is mediated by the cis-gene transcript, i.e., $L_{i} \rightarrow C_{i} \rightarrow T_{j}$. We compared GMAC with other methods in different scenarios, including in the presence of confounders, common child variables, and intermediate variables. A common child variable is a variable that is affected by both $C_{i}$ and $T_{j}$ (Fig. 2C). An intermediate variable is a variable that is affected by $C_{i}$ and affecting $T_{j}$, that is, at least partially mediating the effects from $C_{i}$ to $T_{j}$ (Fig. 2D).

\section{Scenario 1: Under the null in the presence of common child variables}

This is a scenario in which there is one common child variable for each pair of cis- and trans-gene transcripts (Fig. 2C). In this scenario, adjusting for common child variables in mediation analyses would "marry" $C_{i}$ and $T_{j}$ and make $C_{i}$ appear to be regulating $T_{j}$

\section{Genome Research}

www.genome.org 
Table 3. Comparison of the type I error rate and power of GMAC compared to other methods for mediation analysis under the null ( $A$ and $B$ ) and the alternative ( $C$ and $D$ ) hypotheses, based on simulated data

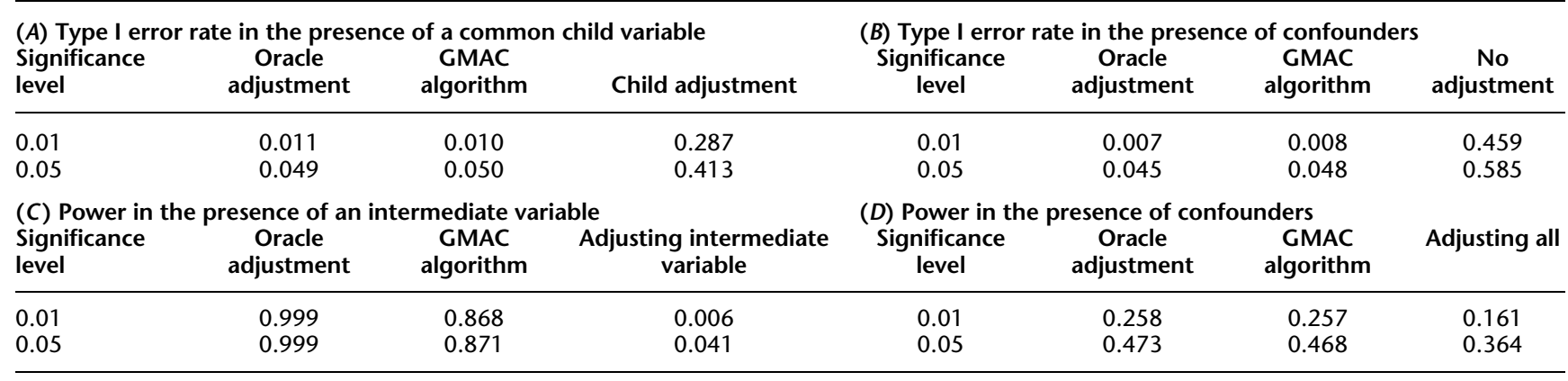

even if there is no such effect (i.e., "collider bias") (Greenland 2003), increasing the false positive rate for detecting mediation. Therefore, we consider it as "improper" to adjust for common child variables. We simulated a pool of independent and normally distributed variables $\mathbf{H}$, with dimensionality being the same as the sample size of 350 . For each of the 1000 mediation trios, we simulated the genetic locus $L_{i}$ under Hardy-Weinberg Equilibrium with a minor allele frequency of 0.1 . Given $L_{i}$, the cis-gene transcript $C_{i}$ and the trans-gene transcript $T_{j}$ are generated according to the models: $C_{i}=\beta_{i 0 c}+\beta_{i 1 c} L_{i}+\varepsilon_{i c}$ and $T_{j}=\beta_{i 0 t}+\beta_{i 1 t} L_{i}+\varepsilon_{i t}$. In this scenario, the trans-effect is not mediated by the cis-gene transcript. We let the parameters in the above models vary across the 1000 trios, with $\beta_{i 1 c}$ sampled uniformly from 0.5 to 1.5 , and the rest sampled uniformly from 0.5 to 1.0 . The error terms $\varepsilon_{i c}$ and $\varepsilon_{i t}$ are normally distributed. For each mediation trio, one candidate variable in $\mathbf{H}$ is randomly chosen to be the common child variable, $Z_{j}$, and the effects of cis- and trans-gene transcripts on $Z_{j}$ are sampled uniformly from 1 to 1.5 .

\section{Scenario 2: Under the null in the presence of confounders}

Scenario 2 is generated under the null in the presence of confounders (Fig. 2B). Each candidate confounding variable has a $5 \%$ probability of being a true confounder of the cis-trans relationship for a randomly chosen proportion of trios where the proportion follows a uniform distribution from 0 to 0.2 . This specification results in, on average, 1.85 confounders for each trio in our simulated data. Suppose for the $i^{\text {th }}$ trio there are $n_{i}$ number of variables in $\mathbf{H}$ selected to be confounders; we denote the confounders as $X_{i 1}, \ldots, X_{i n_{i}}$. The cis-gene transcript $C_{i}$ and trans-gene transcript $T_{j}$ are generated according to the regression models $C_{i}=\beta_{i 0 c}+\beta_{i 1 c} L_{i}+\alpha_{i 1} X_{i 1}+\ldots+\alpha_{i n_{i}} X_{i n_{i}}+\epsilon_{i c}$ and $T_{j}=\beta_{i 0 t}+\beta_{i 1 t} L_{i}+\gamma_{i 1} X_{i 1}+\ldots+\gamma_{i n_{i}} X_{i n_{i}}+\epsilon_{i t}$. We let the parameters in the above models vary across the 1000 trios with similar parameter specification as before. In this scenario, there are no cis- to trans-gene mediation effects. Failure to adjust for confounders may induce false positive results, and it is improper to not adjust for confounders.

\section{Scenario 3: Under the alternative in the presence of intermediate variables}

We consider another scenario in which there is one intermediate variable for each cis-trans relationship (Fig. 2D). For each mediation trio, we simulated the genetic locus and the cis-gene transcript as before and further simulated a child variable, $W_{i}$ of the cis-gene transcript. The trans-gene transcript, $T_{j}$ is then simulated to be affected by $W_{i}$, according to $T_{j}=\beta_{i 0 t}+\beta_{i 1 t} L_{i}+\gamma_{i} W_{i}+\varepsilon_{i t}$. Note that the trans-gene $T_{j}$ is simulated to be affected by the cis-gene $C_{i}$ only via the intermediate variable $W_{i}$. The mediation effects from cis- to trans-gene transcript (via $W_{i}$ ) is nonzero in this scenario. Because $W_{i}$ is on the causal pathway from $C_{i}$ to $T_{j}$, it is improper to adjust for $W_{i}$, and the adjustment will reduce or eliminate power to detect true mediation.

\section{Scenario 4: Under the alternative in the presence of confounders}

To compare with the existing approach that adjusts for a universal set of variables, we consider a scenario in which the dimensionality of potential confounding variables $\mathbf{H}$ is 100 . For each trio, up to five variables in $\mathbf{H}$ are randomly selected to confound the cis-trans gene relationship. The absolute effects of confounders on cis transcripts are sampled uniformly from 0.15 to 0.5 with a $50 \%$ probability to be negative; the effects of confounders on trans transcripts are sampled uniformly from 0.15 to 0.5 , with all to be positive. We set the effect of cis transcript on trans transcript to be 0.1 , i.e., nonzero mediation effects. When the number of potential confounding variables is large, although one may still adjust them all for the simulated sample size, this adjustment is inefficient and may hurt the power.

\section{Simulation results}

For each scenario, we compared the results based on the following methods: (1) Oracle adjustment, which correctly adjusts for the true confounders but no child or intermediate variables in the mediation test; (2) the GMAC algorithm; and (3) improper (or inefficient) adjustment, which corresponds to incorrectly adjusting for the common child variables in scenario 1, failure to adjust for confounders in scenario 2, incorrectly adjusting for the intermediate variables in scenario 3 , and universally adjusting for all variables in $\mathbf{H}$ in scenario 4 , including variables which are not true confounders. Table 3, A and B, shows the true type I error rates at the significance levels of 0.01 and 0.05 in scenarios 1 and 2, respectively. As expected, adjusting for child variables "marries" the cisand trans-genes in the mediation test, resulting in inflated rates of false positive findings. Failure to adjust for confounding also leads to inflated type I error rates. In contrast, both the Oracle and GMAC adjustment control the type I error rates. Table 3C shows that, when the power to detect mediation is high (by Oracle and GMAC), incorrectly adjusting for an intermediate variable in this setting greatly reduces the power to detect mediation. In comparison, GMAC correctly filters out most of the true intermediate variables in the adjustment for mediation tests and maintains power comparable to Oracle adjustment. Table 3D shows that GMAC has 
comparable power to Oracle adjustment in scenario 4. In our simulation, 2962 out of 3023 generated confounders across the 1000 mediation trios are correctly selected. In comparison, adjusting for all variables in the pool of confounders is inefficient and reduces power to detect mediation.

\section{Discussion}

In this work, we have developed the GMAC algorithm for conducting mediation analysis to identify cis transcripts that mediate the effects of trans-eQTLs on distant genes. We address a central problem in mediation analysis, "mediator-outcome confounding," by developing an algorithm that can (1) search a very large pool of variables (surrogate and/or measured) for variables likely to have confounding effects and (2) adaptively adjust for such variables in each mediation test conducted. We acknowledge that we cannot make definitive causal claims regarding any of the mediation/regulatory relationships for which we detect evidence. Instead, the focus of our work is to strengthen the evidence that mediation analysis can provide and to identify candidate cis-hub genes likely to mediate the effects of eQTLs on many trans-genes within or across human tissue types. Cis-hub genes are likely to be key players in the regulatory networks relevant to human disease, thus it is important that we understand their patterns of regulation. By applying GMAC to 44 human tissues from the GTEx project, we are able to characterize cis-hubs with potential disease relevance by aggregating information across many different tissue types. Analyses of simulated data show that the GMAC algorithm improves the power to detect true mediation compared with existing methods, while controlling the true false positive rate.

In analyses of GTEx data, over $20 \%$ of cis-mediators we observe appear to mediate the effects of a trans-eQTL on multiple genes, but the vast majority of these cis-hubs are either tissue-specific (i.e., mediating multiple trans-genes in a single tissue type) or have unique trans targets in each tissue type. We provided one example of a biologically plausible multitissue cis-hub, whereby a cismediator of trans-eQTLs appears to have common trans targets across multiple tissue types. The cis-hub identified (IFI44L) has potential relevance for neurological and psychological disorders, particularly those with etiologies related to immune function, demonstrating the potential value of our approach for understanding disease-relevant pathways.

One innovative aspect of this work is our algorithm that rigorously addresses the problem of "mediator-outcome confounding" in the context of genomic mediation analysis. In eQTLbased mediation analysis, potential confounders of the cis-trans association include demographic and environmental factors, as well as a wide array of biological phenomena, such as expression of specific genes or other biological processes that may be represented by the expression of sets of genes. Neglecting to control for such confounding variables can lead to substantial bias in estimates of mediation, resulting in spurious findings, as we have described previously (Pierce et al. 2014). Considering the complexity of the biological systems under study, as well as the diversity of the GTEx donors, a careful control for such confounding variables is critically important.

Most existing methods control for confounding variables by constructing a set of variables that represent the largest components of variation in the transcriptome and adjusting for the selected set for all tests conducted. However, only when a variable is causally related to both the cis- and trans-genes (as shown in Fig. 2B) will the variable potentially show confounding effects in mediation analysis. GMAC adaptively selects a set of confounding variables for each trio undergoing mediation analysis, enabling large-scale genomic mediation analyses adjusting only for the confounding variables that could potentially bias a specific mediation estimate. As opposed to adjusting for all known covariates, our strategy of selecting only potential confounders for adjustment purposes is important for three reasons: (1) Adjusting for fewer variables increases power (i.e., fewer degrees of freedom) (see Supplemental Table S1); (2) the number of variables from which one selects covariates could be extremely large (e.g., all expressed genes), making adjustment for all covariates impossible; and (3) inadvertently adjusting for "common child" or intermediate variables can result in substantial biases. In this work, we select potential confounders from all expression PCs, but one could also select from among transcripts that are not well-represented by PCs. By efficiently selecting confounders from a very large pool of potential variables, GMAC improves both power and precision in mediation analyses.

There are several limitations of our approach and its application to GTEx data. First, when working with real genomic data, we can never be sure that we have measured and accounted for all possible mediator-outcome confounding. Potential confounders include participant characteristics, environmental factors, and tissue micro-environmental factors, as well as a wide array of biological factors which may or may not be captured by the expression data being analyzed. Second, in the analysis presented here, we only consider the trios with both strong cis- and trans-eQTL effects. For any given tissue type we are analyzing, our sample size is too small for robust genome-wide detection/analysis of transeQTLs. As such, the mediation trios we considered are only a subset of the true mediation trios in the genome, and the small sample sizes may also result in underpowered mediation tests. As the sample size of GTEx increases, future studies will have increased power to identify cis-mediators using GMAC. Third, we did not consider the full complexity of gene isoforms and splice variants in this work; future studies should consider the possibility of mediation relationships that are isoform-specific. Lastly, some trans-eQTLs may not be mediated by variation in the expression of a cis-gene. Other potential mediating mechanisms could include variation in coding sequence, physical inter-chromosomal interaction, or variation in noncoding RNA. Our work is not intended to identify and analyze such trans-eQTLs, as we perform trans-eQTL analyses using only SNPs known to be cis-eSNPs.

It is important to note that our expectation is that most transeQTLs are fully mediated by a transcript that is regulated in cis by the causal trans-eQTL variant. We did not observe "complete mediation" (i.e., $\%$ mediation $=100 \%$ ) for the majority of the significant mediation $P$-values we observed. However, as we have explained and demonstrated previously (Pierce et al. 2014), full mediation will be observed as partial mediation in the presence of mediator measurement error and/or imperfect LD between the causal variant and the variant used for analysis purposes. Thus, considering RNA quantification is not error-free and causal variants are often unknown, we expect to often observe partial mediation when full mediation is present.

We also demonstrate that it is critical to consider mappability for both cis- and trans-genes involved in mediation analysis. For genes containing sequences that do not uniquely map to the human transcriptome, it is possible that gene expression measures may be comprised of signals coming from multiple genes, which can produce false positives in mediation analysis, including spurious detection of cis-hubs and cross-tissue cis-mediators.

\section{Genome Research}

www.genome.org 
Our application of the GMAC algorithm to the multitissue expression data from GTEx provides a unique cross-tissue perspective on cis-mediation of trans-regulatory relationships across human tissues. This multitissue perspective is important because observing mediation relationships that are consistent across multiple tissues provides confidence that a significant mediation $P$-value reflects a true instance of mediation. For the "cis-hub" genes and genes that appear to be cis-mediators in multiple tissues, further investigation is warranted, as these genes may have many regulatory relationships that we are not powered to detect in this work. Thus, a multitissue mediation analysis approach has the potential to increase power to identify true mediators while controlling for false positives. In future work, attempts at joint analyses of multiple tissue types may provide a more complete picture of the cross-tissue and tissue-specific trans-regulatory mechanisms. The GMAC approach described here will be a valuable tool for such studies as well as any future studies that aim to understand the relationships among cis- and trans-eQTLs and characterize the biological mechanisms and networks involved in human disease biology.

We have developed an R GMAC package to perform the proposed genomic mediation analysis with adaptive selection of confounding variables. The package is currently available through $\mathrm{R}$ CRAN.

\section{Methods}

\section{Biospecimen collection and processing of GTEx data}

A total of 7051 tissues samples were obtained from 44 distinct tissue types from 449 post-mortem tissue donors (with $65.6 \%$ male). Those donors were from multiple ethnicity groups, spanned a wide age range, and have various causes of death (see GTEx portal for descriptive statistics). Donor enrollment, consent processes, and biospecimen collection and processing have been described previously (Lonsdale et al. 2013; Ardlie et al. 2015). Briefly, each tissue sample was preserved in a PAXgene tissue kit and stored as both frozen and paraffin-embedded tissue. Total RNA was isolated from PAXgene fixed tissue samples using the PAXgene Tissue mRNA kit. For whole blood, total RNA was isolated from samples collected and preserved in PAXgene blood RNA tubes.

Blood samples were used as the primary source of DNA. Genotyping was conducted using the Illumina Human Omni5Quad and Infinium ExomeChip arrays. Standard QC procedures were performed using PLINK software (Purcell et al. 2007), and genotype imputation was performed using IMPUTE2 software (Howie et al. 2009) and reference haplotypes from the 1000 Genomes Project (The 1000 Genomes Project Consortium 2015). The first three PCs representing ancestry (Price et al. 2006) were included as covariates in all analyses.

RNA-seq data were generated for RNA samples with an RIN value of six or greater. Nonstrand-specific RNA sequencing was performed using an automated version of the Illumina TruSeq RNA sample preparation protocol. Sequencing was done on an Illumina HiSeq 2000, to a median depth of 78M 76-bp pairedend reads per sample. RNA-seq data were aligned to the human genome using TopHat (Trapnell et al. 2009). Gene-level expression was estimated in RPKM units using RNA-SeQC (DeLuca et al. 2012). RNA-seq expression samples that passed various quality control measures (as previously described) were included in the final analysis data set.

\section{Mappability of transcripts}

Because nonuniquely mapping reads can result in false positive eQTLs, we use the mappability of each gene as a quality control filter, as described by The GTEx Consortium (2017). The mappability was calculated as follows: Mappability of all $k$-mers in the reference human genome (hg19) computed by ENCODE (The ENCODE Project Consortium 2012) was downloaded from the UCSC Genome Browser (accession: wgEncodeEH000318, wgEncodeEH00032) (Rosenbloom et al. 2013). The exon- and UTR-mappability of a gene were computed as the average mappability of all $k$-mers in exons and UTRs, respectively. We used $k=$ 75 for exonic regions, as it is the closest to GTEx read length among all possible $k^{\prime}$ s. UTRs are generally quite small, so $k=36$ was used, the smallest among all possible $k$ 's. Mappability of a gene was computed as the weighted average of its exonmappability and UTR-mappability, with the weights being proportional to the total length of exonic regions and UTRs, respectively.

\section{The selection of trios for mediation tests}

In the mediation analysis presented in this work, we consider only the trios with evidence of cis and trans associations, $L_{i} \rightarrow C_{i}$ and $L_{i} \rightarrow T_{j}$. The identification of cis-eQTLs is described elsewhere (Ardlie et al. 2015; The GTEx Consortium 2017). For genes with multiple cis-eSNPs as eQTLs, only one cis-eSNP for each gene (i.e., the high-quality SNP with the smallest $P$-value) was selected and was included in the subsequent trans-eQTL and mediation analyses. The complete cis-eQTL list is available through the GTEx portal (https://gtexportal.org/), and all data can be obtained through dbGaP (phs000424.v6.p1).

Furthermore, using Matrix eQTL (Shabalin 2012), we conducted genome-wide trans-eQTL analyses, restricted to the ciseSNPs described above and examining association for all genes located at least $1 \mathrm{Mb}$ away from the cis-eSNPs. Up to 35 PEER factors and other covariates were adjusted. In each tissue type, when a trans-association $P$-value is less than $10^{-5}$, the eSNP, its corresponding cis transcript, and the trans transcript were treated as a candidate trio and were retained for mediation analysis.

\section{The GMAC algorithm}

In order to identify cis-mediators of trans-eQTLs across the genome, we propose the GMAC algorithm (Fig. 2A). Here, we present a brief description of each step. A detailed description and justification for each can be found in the Supplemental Methods. Specifically,

- Step 0. We focus on only the trios $\left(L_{i}, C_{i}, T_{j}\right)$ in the genome showing both cis- and trans-eQTL associations, i.e., $L_{i} \rightarrow C_{i}$ and $L_{i} \rightarrow T_{j}$. Consider a pool of candidate variables $\mathbf{H}$ consisting of either real covariates, constructed surrogate variables, or both.

- Step 1. Filter out common child and intermediate variables from the pool of potential confounders. For each trio $\left(L_{i}, C_{i}, T_{j}\right)$, we calculate the marginal associations of variables in $\mathbf{H}$ to $L_{i}$ and filter the ones with significant associations at the 10\% FDR level. As shown in Figure 2B-D, common child and intermediate variables are directly associated with $L_{i}$, while confounders are assumed to be unassociated with $L_{i}$. Note that since genetic loci are "Mendelian randomized" (Smith and Ebrahim 2003), without loss of generality we assume the confounders are not associated with $L_{i}$. Let $\mathbf{H}_{\mathbf{i j}}$ denote the retained pool of candidate variables specific to the trio $\left(L_{i}, C_{i}, T_{j}\right)$.

- Step 2. Adaptively select confounders. For each trio and each of its potential confounding variables in $\mathbf{H}_{\mathbf{i j}}$, we calculate the $P$ value of the overall $F$-test to assess the association of the variable to at least one of the cis and trans transcripts. Considering the $P$-values for one potential confounding variable to all trios 
as one stratum, we apply a $10 \%$ FDR significance threshold to each stratum (each column in Fig. 2E) - a stratified FDR approach (Sun et al. 2006). The significant variables corresponding to a trio (each row in Fig. 2E) will be selected in the mediation analyses as the adaptively selected confounders specific to that trio (see Supplemental Methods for details). Let $\mathbf{X}_{\mathbf{i j}}$ denote the list of adaptively selected confounding variables for the trio, $\left(L_{i}, C_{i}, T_{j}\right)$.

- Step 3. Test for mediation. For each trio and its adaptively selected confounder set, we calculate the mediation statistic as the Wald statistic for testing the indirect mediation effect $H_{0}: \beta_{1}=$ $O$ based on the following regression regressing the trans-gene expression levels on the cis-gene expression levels adjusting for the cis-eQTL, other covariates, and selected confounders:

$$
T_{j}=\beta_{0}+\beta_{1} C_{i}+\beta_{2} L_{i}+\Gamma X_{i j}+\varepsilon .
$$

We perform within-genotype group permutation on the cisgene transcript at least 10,000 times and recalculate each null mediation statistic based on the locus, a permuted cis-gene transcript, and the trans-gene transcript, $\left(L_{i}, C_{i 0}, T_{j}\right)$. Figure $2 \mathrm{~F}$ shows the expression variation patterns of a hypothetical mediation relationship $L_{i} \rightarrow C_{i} \rightarrow T_{j}$ on the left panel, and a null relationship entailed by $\left(L_{i}, C_{i 0}, T_{j}\right)$ with $L_{i} \rightarrow C_{i 0}$ and $L_{i} \rightarrow T_{j}$ but no mediation. It justifies that by permuting the cis-gene expression levels within each genotype group, one maintains the cisassociations while breaking the potential mediation effects from the cis- to the trans-gene transcript (i.e., conditional correlations of cis and trans transcript conditioning on $L_{i}$, or correlation within each genotype group). We calculate the $P$-value of mediation for the trio $\left(L_{i}, C_{i}, T_{j}\right)$ by comparing the observed mediation statistic with the null statistics.

The proposed algorithm is superior to existing approaches for mediation analysis that adjust a universal set of variables for all trios. GMAC avoids the adjustment of common child variables, intermediate variables, and unrelated variables in genomic mediation analysis, and it is able to search a much larger pool of variables for potential confounders, not just those captured by the top few surrogate variables or PCs.

\section{Software availability}

The R software package GMAC is available in the Supplemental Materials and also online through R CRAN https://cran.r-project. org/.

\section{GTEx Consortium}

\section{Laboratory, Data Analysis \& Coordinating Center (LDACC)- Analysis Working Group}

François Aguet, ${ }^{6}$ Kristin G. Ardlie, ${ }^{6}$ Beryl B. Cummings, ${ }^{6,7}$ Ellen T. Gelfand, ${ }^{6}$ Gad Getz, ${ }^{6,8}$ Kane Hadley, ${ }^{6}$ Robert E. Handsaker, ${ }^{6,9}$ Katherine H. Huang, ${ }^{6}$ Seva Kashin, ${ }^{6,9}$ Konrad J. Karczewski, ${ }^{6,7}$ Monkol Lek, ${ }^{6,7}$ Xiao Li, ${ }^{6}$ Daniel G. MacArthur, ${ }^{6,7}$ Jared L. Nedzel, ${ }^{6}$ Duyen T. Nguyen, ${ }^{6}$ Michael S. Noble, ${ }^{6}$ Ayellet V. Segrè, ${ }^{6}$ Casandra A. Trowbridge, ${ }^{6}$ and Taru Tukiainen ${ }^{6,7}$

\footnotetext{
${ }^{6}$ The Broad Institute of Massachusetts Institute of Technology and Harvard University, Cambridge, MA 02142, USA

${ }^{7}$ Analytic and Translational Genetics Unit, Massachusetts General Hospital, Boston, MA 02114, USA

${ }^{8}$ Massachusetts General Hospital Cancer Center and Dept. of Pathology, Massachusetts General Hospital, Boston, MA 02114, USA

${ }^{9}$ Department of Genetics, Harvard Medical School, Boston, MA 02114, USA
}

\section{Statistical Methods groups-Analysis Working Group}

Nathan S. Abell, ${ }^{10,11}$ Brunilda Balliu, ${ }^{11}$ Ruth Barshir, ${ }^{12}$ Omer Basha, $^{12}$ Alexis Battle, ${ }^{13}$ Gireesh K. Bogu, ${ }^{14,15}$ Andrew Brown, ${ }^{16,17,18}$ Christopher D. Brown, ${ }^{19}$ Stephane E. Castel, ${ }^{20,21}$ Lin S. Chen, ${ }^{22}$ Colby Chiang, ${ }^{23}$ Donald F. Conrad, ${ }^{24,25}$ Nancy J. Cox $^{26}$ Farhan N. Damani, ${ }^{13}$ Joe R. Davis, ${ }^{10,11}$ Olivier Delaneau, ${ }^{16,17,18}$ Emmanouil T. Dermitzakis, ${ }^{16,17,18}$ Barbara E. Engelhardt, ${ }^{27}$ Eleazar Eskin, ${ }^{28,29}$ Pedro G. Ferreira, ${ }^{30,31}$ Laure Frésard, ${ }^{10,11}$ Eric R. Gamazon, ${ }^{26,32,33}$ Diego Garrido-Martín, ${ }^{14,15}$ Ariel D.H. Gewirtz, ${ }^{34}$ Genna Gliner, ${ }^{35}$ Michael J. Gloudemans, ${ }^{10,11,36}$ Roderic Guigo, ${ }^{14,15,37}$ Ira M. Hall, ${ }^{23,24,38}$ Buhm Han, ${ }^{39}$ Yuan $\mathrm{He}^{40}$ Farhad Hormozdiari, ${ }^{28}$ Cedric Howald, ${ }^{16,17,18}$ Hae Kyung Im, ${ }^{41}$ Brian Jo, ${ }^{34}$ Eun Yong Kang, ${ }^{28}$ Yungil Kim, ${ }^{13}$ Sarah Kim-Hellmuth, ${ }^{20,21}$ Tuuli Lappalainen, ${ }^{20,21}$

${ }^{10}$ Department of Genetics, Stanford University, Stanford, CA 94305, USA

${ }^{11}$ Department of Pathology, Stanford University, Stanford, CA 94305, USA

${ }^{12}$ Department of Clinical Biochemistry and Pharmacology, Faculty of Health Sciences, Ben-Gurion University of the Negev, Beer-Sheva 84105, Israel

${ }^{13}$ Department of Computer Science, Johns Hopkins University, Baltimore, MD 21218, USA

${ }^{14}$ Centre for Genomic Regulation (CRG), The Barcelona Institute for Science and Technology, 08003 Barcelona, Spain

${ }^{15}$ Universitat Pompeu Fabra (UPF), 08003 Barcelona, Spain

${ }^{16}$ Department of Genetic Medicine and Development, University of Geneva Medical School, 1211 Geneva, Switzerland

${ }^{17}$ Institute for Genetics and Genomics in Geneva (iG3), University of Geneva, 1211 Geneva, Switzerland

${ }^{18}$ Swiss Institute of Bioinformatics, 1211 Geneva, Switzerland

${ }^{19}$ Department of Genetics, Perelman School of Medicine, University of Pennsylvania, Philadelphia, PA 19104, USA

${ }^{20}$ New York Genome Center, New York, NY 10013, USA

${ }^{21}$ Department of Systems Biology, Columbia University Medical Center, New York, NY 10032, USA

${ }^{22}$ Department of Public Health Sciences, The University of Chicago, Chicago, IL 60637, USA

${ }^{23} \mathrm{McD}$ onnell Genome Institute, Washington University School of Medicine, St. Louis, MO 63108, USA

${ }^{24}$ Department of Genetics, Washington University School of Medicine, St. Louis, MO 63108, USA

${ }^{25}$ Department of Pathology \& Immunology, Washington University School of Medicine, St. Louis, MO 63108, USA

${ }^{26}$ Division of Genetic Medicine, Department of Medicine, Vanderbilt University Medical Center, Nashville, TN 37232, USA

${ }^{27}$ Department of Computer Science, Center for Statistics and Machine Learning, Princeton University, Princeton, NJ 08540, USA

${ }^{28}$ Department of Computer Science, University of California, Los Angeles, CA 90095, USA

${ }^{29}$ Department of Human Genetics, University of California, Los Angeles, CA 90095, USA

${ }^{30}$ Instituto de Investigação e Inovação em Saúde (i3S), Universidade do Porto, 4200-135 Porto, Portugal

${ }^{31}$ Institute of Molecular Pathology and Immunology (IPATIMUP), University of Porto, 4200-625 Porto, Portugal

${ }^{32}$ Department of Clinical Epidemiology, Biostatistics and Bioinformatics, Academic Medical Center, University of Amsterdam, 1105 AZ Amsterdam, The Netherlands

${ }^{33}$ Department of Psychiatry, Academic Medical Center, University of Amsterdam, 1105 AZ Amsterdam, The Netherlands

${ }^{34}$ Lewis Sigler Institute, Princeton University, Princeton, NJ 08540, USA

${ }^{35}$ Department of Operations Research and Financial Engineering, Princeton University, Princeton, NJ 08540, USA

${ }^{36}$ Biomedical Informatics Program, Stanford University, Stanford, CA 94305, USA

${ }^{37}$ Institut Hospital del Mar d'Investigacions Mèdiques (IMIM), 08003 Barcelona, Spain

${ }^{38}$ Department of Medicine, Washington University School of Medicine, St. Louis, MO 63108, USA.

${ }^{39}$ Department of Convergence Medicine, University of Ulsan College of Medicine, Asan Medical Center, Seoul 138-736, South Korea

${ }^{40}$ Department of Biomedical Engineering, Johns Hopkins University, Baltimore, MD 21218, USA

${ }^{41}$ Section of Genetic Medicine, Department of Medicine, The University of Chicago, Chicago, IL 60637, USA 
Gen Li, ${ }^{42}$ Xin Li, ${ }^{11}$ Boxiang Liu, ${ }^{10,11,43}$ Serghei Mangul, ${ }^{28}$ Mark I. McCarthy, ${ }^{44,45,46}$ Ian C. McDowell, ${ }^{47}$ Pejman Mohammadi, ${ }^{20,21}$ Jean Monlong, ${ }_{14,15,48}$ Stephen B. Montgomery, ${ }^{10,11}$ Manuel Muñoz-Aguirre, ${ }^{14,15,49}$ Anne W. Ndungu, ${ }^{44}$ Dan L. Nicolae, ${ }^{41,50,51}$ Andrew B. Nobel, ${ }^{52,53}$ Meritxell Oliva, ${ }^{41,54}$ Halit Ongen, ${ }^{16,17,18}$ John J. Palowitch, ${ }^{52}$ Nikolaos Panousis, ${ }^{16,17,18}$ Panagiotis Papasaikas, ${ }^{14,15}$ YoSon Park, ${ }^{19}$ Princy Parsana, ${ }^{13}$ Anthony J. Payne, ${ }^{44}$ Christine B. Peterson, ${ }^{55}$ Jie Quan, ${ }^{56}$ Ferran Reverter, ${ }^{14,15,57}$ Chiara Sabatti, ${ }^{58,59}$ Ashis Saha, ${ }^{13}$ Michael Sammeth, ${ }^{60}$ Alexandra J. Scott, ${ }^{23}$ Andrey A. Shabalin, ${ }^{61}$ Reza Sodaei, ${ }^{14,15}$ Matthew Stephens, ${ }^{50,51}$ Barbara E. Stranger, ${ }^{41,54,62}$ Benjamin J. Strober, ${ }^{40}$ Jae Hoon Sul, ${ }^{63}$ Emily K. Tsang, ${ }^{11,36}$ Sarah Urbut $^{51}$ Martijn van de Bunt, ${ }^{44,45}$ Gao Wang, ${ }^{51}$ Xiaoquan Wen, ${ }^{64}$ Fred A. Wright, ${ }^{65}$ Hualin S. Xi ${ }^{56}$ Esti Yeger-Lotem, ${ }^{12,66}$ Zachary Zappala, ${ }^{10,11}$ Judith B. Zaugg, ${ }^{67}$ and Yi-Hui Zhou ${ }^{65}$

\section{Enhancing GTEx (eGTEx) groups}

Joshua M. Akey, ${ }^{34,68}$ Daniel Bates, ${ }^{69}$ Joanne Chan, ${ }^{10}$ Lin S. Chen, ${ }^{22}$ Melina Claussnitzer, ${ }^{6,70,71}$ Kathryn Demanelis, ${ }^{22}$ Morgan Diegel, ${ }^{69}$ Jennifer A. Doherty, ${ }^{72}$ Andrew P. Feinberg, ${ }^{40,73,74,75}$

${ }^{42}$ Department of Biostatistics, Mailman School of Public Health, Columbia University, New York, NY 10032, USA

${ }^{43}$ Department of Biology, Stanford University, Stanford, CA 94305, USA

${ }^{44}$ Wellcome Trust Centre for Human Genetics, Nuffield Department of Medicine, University of Oxford, Oxford, OX3 7BN, UK

${ }^{45}$ Oxford Centre for Diabetes, Endocrinology and Metabolism, University of Oxford, Churchill Hospital, Oxford, OX3 7LE, UK

${ }^{46}$ Oxford NIHR Biomedical Research Centre, Churchill Hospital, Oxford, OX3 7LJ, UK

${ }^{47}$ Computational Biology \& Bioinformatics Graduate Program, Duke University, Durham, NC 27708, USA

${ }^{48}$ Human Genetics Department, McGill University, Montreal, Quebec H3A 0G1, Canada

${ }^{49}$ Departament d'Estadística i Investigació Operativa, Universitat Politècnica de Catalunya, 08034 Barcelona, Spain

${ }^{50}$ Department of Statistics, The University of Chicago, Chicago, IL 60637, USA ${ }^{51}$ Department of Human Genetics, The University of Chicago, Chicago, IL 60637, USA

${ }^{52}$ Department of Statistics and Operations Research, University of North Carolina, Chapel Hill, NC 27599, USA

${ }^{53}$ Department of Biostatistics, University of North Carolina, Chapel Hill, NC 27599, USA

${ }^{54}$ Institute for Genomics and Systems Biology, The University of Chicago, Chicago, IL 60637, USA

${ }^{55}$ Department of Biostatistics, The University of Texas MD Anderson Cancer Center, Houston, TX 77030, USA

${ }^{56}$ Computational Sciences, Pfizer Inc, Cambridge, MA 02139, USA

${ }^{57}$ Universitat de Barcelona, 08028 Barcelona, Catalonia, Spain

${ }^{58}$ Department of Biomedical Data Science, Stanford University, Stanford, CA 94305, USA

${ }^{59}$ Department of Statistics, Stanford University, Stanford, CA 94305, USA

${ }^{60}$ Institute of Biophysics Carlos Chagas Filho (IBCCF), Federal University of Rio de Janeiro (UFRJ), 21941902 Rio de Janeiro, Brazil

${ }_{62}^{61}$ Department of Psychiatry, University of Utah, Salt Lake City, UT 84108, USA ${ }^{62}$ Center for Data Intensive Science, The University of Chicago, Chicago, IL 60637, USA

${ }^{63}$ Department of Psychiatry and Biobehavioral Sciences, University of California, Los Angeles, CA 90095, USA

${ }^{64}$ Department of Biostatistics, University of Michigan, Ann Arbor, MI 48109, USA

${ }^{65}$ Bioinformatics Research Center and Departments of Statistics and Biological Sciences, North Carolina State University, Raleigh, NC 27695, USA

${ }^{66}$ National Institute for Biotechnology in the Negev, Beer-Sheva, 84105 Israel

${ }^{67}$ European Molecular Biology Laboratory, 69117 Heidelberg, Germany

${ }^{68}$ Department of Ecology and Evolutionary Biology, Princeton University, Princeton, NJ 08540, USA

${ }^{69}$ Altius Institute for Biomedical Sciences, Seattle, WA 98121, USA

${ }^{70}$ Beth Israel Deaconess Medical Center, Harvard Medical School, Boston, MA 02215, USA

${ }^{71}$ University of Hohenheim, 70599 Stuttgart, Germany
Marian S. Fernando, ${ }^{41,54}$ Jessica Halow, ${ }^{69}$ Kasper D. Hansen, ${ }^{73,76,77}$ Eric Haugen, ${ }^{69}$ Peter F. Hickey, ${ }^{77}$ Lei Hou, ${ }^{6,78}$ Farzana Jasmine, ${ }^{22}$ Ruiqi Jian, ${ }^{10}$ Lihua Jiang, ${ }^{10}$ Audra Johnson, ${ }^{69}$ Rajinder Kaul, ${ }^{69}$ Manolis Kellis, ${ }^{6,78}$ Muhammad G. Kibriya, ${ }^{22}$ Kristen Lee, ${ }^{69}$ Jin Billy Li, ${ }^{10}$ Qin Li, ${ }^{10}$ Xiao Li, ${ }^{10}$ Jessica Lin, ${ }^{10,79}$ Shin Lin, ${ }^{10,80}$ Sandra Linder, ${ }^{10,11}$ Caroline Linke, ${ }^{41,54}$ Yaping Liu, ${ }^{6,78}$ Matthew T. Maurano, ${ }^{81}$ Benoit Molinie, ${ }^{6}$ Stephen B. Montgomery, ${ }^{10,11}$ Jemma Nelson, ${ }^{69}$ Fidencio J. Neri, ${ }^{69}$ Meritxell Oliva $^{41,54}$ Yongjin Park, ${ }^{6,78}$ Brandon L. Pierce, ${ }^{22}$ Nicola J. Rinaldi, ${ }^{6,78}$ Lindsay F. Rizzardi, ${ }^{73}$ Richard Sandstrom, ${ }^{69}$ Andrew Skol, ${ }^{41,54,62}$ Kevin S. Smith, ${ }^{10,11}$ Michael P. Snyder, ${ }^{10}$ John Stamatoyannopoulos, ${ }^{69,79,82}$ Barbara E. Stranger, ${ }^{41,54,62}$ Hua Tang, ${ }^{10}$ Emily K. Tsang, ${ }^{11,36}$ Li Wang, ${ }^{6}$ Meng Wang, ${ }^{10}$ Nicholas Van Wittenberghe, ${ }^{6}$ Fan $\mathrm{Wu},{ }^{41,54}$ and Rui Zhang ${ }^{10}$

\section{NIH Common Fund}

Concepcion R. Nierras ${ }^{83}$

\section{$\mathrm{NIH} / \mathrm{NCl}$}

Philip A. Branton, ${ }^{84}$ Latarsha J. Carithers, ${ }^{84,85}$ Ping Guan, ${ }^{84}$ Helen M. Moore, ${ }^{84}$ Abhi Rao, ${ }^{84}$ and Jimmie B. Vaught ${ }^{84}$

\section{$\mathrm{NIH} / \mathrm{NHGRI}$}

Sarah E. Gould, ${ }^{86}$ Nicole C. Lockart, ${ }^{86}$ Casey Martin, ${ }^{86}$ Jeffery P. Struewing, ${ }^{86}$ and Simona Volpi ${ }^{86}$

\section{$\mathrm{NIH} / \mathrm{NIMH}$}

Anjene M. Addington ${ }^{87}$ and Susan E. Koester ${ }^{87}$

\section{NIH/NIDA}

A. Roger Little ${ }^{88}$

${ }^{72}$ Huntsman Cancer Institute, Department of Population Health Sciences, University of Utah, Salt Lake City, UT 84112, USA

${ }^{73}$ Center for Epigenetics, Johns Hopkins University School of Medicine, Baltimore, MD 21205, USA

${ }^{74}$ Department of Medicine, Johns Hopkins University School of Medicine, Baltimore, MD 21205, USA

${ }^{75}$ Department of Mental Health, Johns Hopkins University School of Public Health, Baltimore, MD 21205, USA

${ }^{76}$ McKusick-Nathans Institute of Genetic Medicine, Johns Hopkins School of Medicine, Baltimore, MD 21205, USA

${ }^{77}$ Department of Biostatistics, Johns Hopkins University, Baltimore, MD 21205, USA

${ }^{78}$ Computer Science and Artificial Intelligence Laboratory, Massachusetts Institute of Technology, Cambridge, MA 02139, USA

${ }^{79}$ Department of Medicine, University of Washington, Seattle, WA 98195, USA ${ }^{80}$ Division of Cardiology, University of Washington, Seattle, WA 98195, USA

${ }^{81}$ Institute for Systems Genetics, New York University Langone Medical Center, New York, NY 10016, USA

${ }^{82}$ Department of Genome Sciences, University of Washington, Seattle, WA 98195, USA

${ }^{83}$ Office of Strategic Coordination, Division of Program Coordination, Planning and Strategic Initiatives, Office of the Director, NIH, Rockville, MD 20852, USA ${ }^{84}$ Biorepositories and Biospecimen Research Branch, Division of Cancer Treatment and Diagnosis, National Cancer Institute, Bethesda, MD 20892, USA ${ }^{85}$ National Institute of Dental and Craniofacial Research, Bethesda, MD 20892, USA

${ }^{86}$ Division of Genomic Medicine, National Human Genome Research Institute, Rockville, MD 20852, USA

${ }^{87}$ Division of Neuroscience and Basic Behavioral Science, National Institute of Mental Health, NIH, Bethesda, MD 20892, USA

${ }^{88}$ Division of Neuroscience and Behavior, National Institute on Drug Abuse, NIH, Bethesda, MD 20892, USA 


\section{Biospecimen Collection Source Site-NDRI}

Lori E. Brigham, ${ }^{89}$ Richard Hasz, ${ }^{90}$ Marcus Hunter, ${ }^{91}$ Christopher Johns, ${ }^{92}$ Mark Johnson, ${ }^{93}$ Gene Kopen, ${ }^{94}$ William F. Leinweber, ${ }^{94}$ John T. Lonsdale, ${ }^{94}$ Alisa McDonald, ${ }^{94}$ Bernadette Mestichelli, ${ }^{94}$ Kevin Myer, ${ }^{91}$ Brian Roe, ${ }^{91}$ Michael Salvatore, ${ }^{94}$ Saboor Shad, ${ }^{94}$ Jeffrey A. Thomas, ${ }^{94}$ Gary Walters, ${ }^{93}$ Michael Washington, ${ }^{93}$ and Joseph Wheeler ${ }^{92}$

\section{Biospecimen Collection Source Site- RPCI}

Jason Bridge, ${ }^{95}$ Barbara A. Foster, ${ }^{96}$ Bryan M. Gillard, ${ }^{96}$ Ellen Karasik, ${ }^{96}$ Rachna Kumar, ${ }^{96}$ Mark Miklos, ${ }^{95}$ and Michael T. Moser $^{96}$

\section{Biospecimen Core Resource-VARI}

Scott D. Jewell, ${ }^{97}$ Robert G. Montroy, ${ }^{97}$ Daniel C. Rohrer, ${ }^{97}$ and Dana R. Valley ${ }^{97}$

\section{Brain Bank Repository-University of Miami Brain Endowment Bank}

David A. Davis ${ }^{98}$ and Deborah C. Mash ${ }^{98}$

\section{Leidos Biomedical—Project Management}

Anita H. Undale, ${ }^{99}$ Anna M. Smith, ${ }^{100}$ David E. Tabor, ${ }^{100}$ Nancy V. Roche, ${ }^{100}$ Jeffrey A. McLean, ${ }^{100}$ Negin Vatanian, ${ }^{100}$ Karna L. Robinson, ${ }^{100}$ Leslie Sobin, ${ }^{100}$ Mary E. Barcus, ${ }^{101}$ Kimberly M. Valentino, ${ }^{100}$ Liqun Qi, ${ }^{100}$ Steven Hunter, ${ }^{100}$ Pushpa Hariharan, ${ }^{100}$ Shilpi Singh, ${ }^{100}$ Ki Sung Um, ${ }^{100}$ Takunda Matose, ${ }^{100}$ and Maria M. Tomaszewski ${ }^{100}$

\section{ELSI Study}

Laura K. Barker, ${ }^{102}$ Maghboeba Mosavel, ${ }^{103}$ Laura A. Siminoff, ${ }^{102}$ and Heather M. Traino ${ }^{102}$

\section{Genome Browser Data Integration \& Visualization-EBI}

Paul Flicek, ${ }^{104}$ Thomas Juettemann, ${ }^{104}$ Magali Ruffier, ${ }^{104}$ Dan Sheppard, ${ }^{104}$ Kieron Taylor, ${ }^{104}$ Stephen J. Trevanion, ${ }^{104}$ and Daniel R. Zerbino ${ }^{104}$

\footnotetext{
${ }^{89}$ Washington Regional Transplant Community, Falls Church, VA 22003, USA ${ }^{90} \mathrm{Gift}$ of Life Donor Program, Philadelphia, PA 19103, USA

${ }^{91}$ LifeGift, Houston, TX 77055, USA

${ }^{92}$ Center for Organ Recovery and Education, Pittsburgh, PA 15238, USA

${ }^{93}$ LifeNet Health, Virginia Beach, VA 23453, USA

${ }^{94}$ National Disease Research Interchange, Philadelphia, PA 19103, USA

${ }^{95}$ Unyts, Buffalo, NY 14203, USA

${ }^{96}$ Pharmacology and Therapeutics, Roswell Park Cancer Institute, Buffalo, NY 14263, USA

${ }^{97}$ Van Andel Research Institute, Grand Rapids, MI 49503, USA

${ }^{98}$ Brain Endowment Bank, Miller School of Medicine, University of Miami, Miami, FL 33136, USA

${ }^{99}$ National Institute of Allergy and Infectious Diseases, NIH, Rockville, MD 20852, USA

${ }^{00}$ Biospecimen Research Group, Clinical Research Directorate, Leidos Biomedical Research, Inc., Rockville, MD 20852, USA

${ }^{101}$ Leidos Biomedical Research, Inc., Frederick, MD 21701, USA

${ }^{102}$ Temple University, Philadelphia, PA 19122, USA

${ }^{103}$ Department of Health Behavior and Policy, School of Medicine, Virginia Commonwealth University, Richmond, VA 23298, USA

${ }^{104}$ European Molecular Biology Laboratory, European Bioinformatics Institute,
} Hinxton CB10 1SD, UK
Genome Browser Data Integration \& Visualization-UCSC Genomics Institute, University of California Santa Cruz

Brian Craft, ${ }^{105}$ Mary Goldman, ${ }^{105}$ Maximilian Haeussler, ${ }^{105}$ W. James Kent, ${ }^{105}$ Christopher M. Lee, ${ }^{105}$ Benedict Paten, ${ }^{105}$ Kate R. Rosenbloom, ${ }^{105}$ John Vivian, ${ }^{105}$ and Jingchun $\mathrm{Zhu}^{105}$

\section{Acknowledgments}

We thank Alexis Battle for providing the estimates of mappability used in this work. The Genotype-Tissue Expression (GTEx) Project was supported by the Common Fund of the Office of the Director of the National Institutes of Health. Additional funds were provided by the NCI, NHGRI, NHLBI, NIDA, NIMH, and NINDS. Donors were enrolled at Biospecimen Source Sites funded by NCI $\backslash$ SAICFrederick, Inc. (SAIC-F) subcontracts to the National Disease Research Interchange (10XS170), Roswell Park Cancer Institute (10XS171), and Science Care, Inc. (X10S172). The Laboratory, Data Analysis, and Coordinating Center (LDACC) was funded through a contract (HHSN268201000029C) to The Broad Institute, Inc. Biorepository operations were funded through an SAIC-F subcontract to Van Andel Institute (10ST1035). Additional data repository and project management were provided by SAIC-F (HHSN261200800001E). The Brain Bank was supported by supplements to University of Miami grants DA006227 and DA033684 and to contract N01MH000028. Statistical Methods development grants were made to the University of Geneva (MH090941 and MH101814), the University of Chicago (MH090951, MH090937, MH101820, MH101825), the University of North Carolina-Chapel Hill (MH090936 and MH101819), Harvard University (MH090948), Stanford University (MH101782), Washington University St. Louis (MH101810), and the University of Pennsylvania (MH101822). The data used for the analyses described in this manuscript were obtained from the GTEx Portal in January of 2015. This work was supported by National Institutes of Health grants R01 GM108711 (NIGMS), U01 HG007601 (NHGRI), and R01 MH101820 (NIMH).

\section{References}

The 1000 Genomes Project Consortium. 2015. A global reference for human genetic variation. Nature 526: $68-74$.

Ardlie KG, DeLuca DS, Segre AV, Sullivan TJ, Young TR, Gelfand ET, Trowbridge CA, Maller JB, Tukiainen T, Lek M, et al. 2015. The Genotype-Tissue Expression (GTEx) pilot analysis: multitissue gene regulation in humans. Science 348: 648-660.

Battle A, Mostafavi S, Zhu XW, Potash JB, Weissman MM, McCormick C, Haudenschild CD, Beckman KB, Shi JX, Mei R, et al. 2014 Characterizing the genetic basis of transcriptome diversity through RNA-sequencing of 922 individuals. Genome Res 24: 14-24.

Chen LS, Emmert-Streib F, Storey JD. 2007. Harnessing naturally randomized transcription to infer regulatory relationships among genes. Genome Biol 8: R219.

Chen DT, Jiang X, Akula N, Shugart YY, Wendland JR, Steele CJ, Kassem L, Park JH, Chatterjee N, Jamain S, et al. 2013. Genome-wide association study meta-analysis of European and Asian-ancestry samples identifies three novel loci associated with bipolar disorder. Mol Psychiatry 18: 195-205.

Cheon H, Stark GR. 2009. Unphosphorylated STAT1 prolongs the expression of interferon-induced immune regulatory genes. Proc Natl Acad Sci 106: 9373-9378.

Cole SR, Hernan MA. 2002. Fallibility in estimating direct effects. Int J Epidemiol 31: 163-165

${ }^{105}$ UCSC Genomics Institute, University of California Santa Cruz, Santa Cruz, CA 95064, USA 
DeLuca DS, Levin JZ, Sivachenko A, Fennell T, Nazaire MD, Williams C, Reich M, Winckler W, Getz G. 2012. RNA-SeQC: RNA-seq metrics for quality control and process optimization. Bioinformatics 28: 1530-1532.

The ENCODE Project Consortium. 2012. An integrated encyclopedia of DNA elements in the human genome. Nature 489: 57-74.

Feenstra B, Pasternak B, Geller F, Carstensen L, Wang T, Huang F, Eitson JL, Hollegaard MV, Svanstrom H, Vestergaard M, et al. 2014. Common variants associated with general and MMR vaccine-related febrile seizures. Nat Genet 46: 1274-1282.

Fehrmann RSN, Jansen RC, Veldink JH, Westra HJ, Arends D, Bonder MJ, Fu JY, Deelen P, Groen HJM, Smolonska A, et al. 2011. Trans-eQTLs reveal that independent genetic variants associated with a complex phenotype converge on intermediate genes, with a major role for the HLA. PLoS Genet 7: e1002197.

Greenland S. 2003. Quantifying biases in causal models: classical confounding vs collider-stratification bias. Epidemiology 14: 300-306.

The GTEx Consortium. 2017. Genetic effects on gene expression across human tissues. Nature 550: 204-213.

Howie BN, Donnelly P, Marchini J. 2009. A flexible and accurate genotype imputation method for the next generation of genome-wide association studies. PLoS Genet 5: e1000529.

Ioannidis I, McNally B, Willette M, Peeples ME, Chaussabel D, Durbin JE, Ramilo O, Mejias A, Flano E. 2012. Plasticity and virus specificity of the airway epithelial cell immune response during respiratory virus infection. J Virol 86: 5422-5436.

Kompass KS, Witte JS. 2011. Co-regulatory expression quantitative trait loci mapping: method and application to endometrial cancer. BMC Med Genomics 4: 6 .

Kyogoku C, Smiljanovic B, Grun JR, Biesen R, Schulte-Wrede U, Haupl T, Hiepe F, Alexander T, Radbruch A, Grutzkau A. 2013. Cell-specific type I IFN signatures in autoimmunity and viral infection: what makes the difference? PLoS One 8: e83776.

Leek JT, Storey JD. 2007. Capturing heterogeneity in gene expression studies by surrogate variable analysis. PLoS Genet 3: 1724-1735.

Lonsdale J, Thomas J, Salvatore M, Phillips R, Lo E, Shad S, Hasz R, Walters G, Garcia F, Young N, et al. 2013. The Genotype-Tissue Expression (GTEx) project. Nat Genet 45: 580-585.

Nicolae DL, Gamazon E, Zhang W, Duan SW, Dolan ME, Cox NJ. 2010. Trait-associated SNPs are more likely to be eQTLs: annotation to enhance discovery from GWAS. PLoS Genet 6: e1000888.

Pearl J. 2001. Direct and indirect effects. In Proc. of the seventeenth conference in Uncertainty in Artificial Intelligence, pp. 411-420. Morgan Kaufmann, San Francisco, CA.

Pierce BL, Tong L, Chen LS, Rahaman R, Argos M, Jasmine F, Roy S, PaulBrutus R, Westra HJ, Franke L, et al. 2014. Mediation analysis demonstrates that trans-eQTLs are often explained by cis-mediation: a genome-wide analysis among 1,800 South Asians. PLoS Genet 10: e1004818.

Price AL, Patterson NJ, Plenge RM, Weinblatt ME, Shadick NA, Reich D. 2006. Principal components analysis corrects for stratification in genome-wide association studies. Nat Genet 38: 904-909.

Purcell S, Neale B, Todd-Brown K, Thomas L, Ferreira MAR, Bender D, Maller J, Sklar P, de Bakker PIW, Daly MJ, et al. 2007. PLINK: a tool set for whole-genome association and population-based linkage analyses. Am J Hum Genet 81: 559-575.

Robins JM, Greenland S. 1992. Identifiability and exchangeability for direct and indirect effects. Epidemiology 3: 143-155.

Rosenbloom KR, Sloan CA, Malladi VS, Dreszer TR, Learned K, Kirkup VM, Wong MC, Maddren M, Fang R, Heitner SG, et al. 2013. ENCODE data in the UCSC Genome Browser: year 5 update. Nucleic Acids Res 41: D56-D63.

Ruderfer DM, Fanous AH, Ripke S, McQuillin A, Amdur RL, Gejman PV, O'Donovan MC, Andreassen OA, Djurovic S, Hultman CM, et al. 2014. Polygenic dissection of diagnosis and clinical dimensions of bipolar disorder and schizophrenia. Mol Psychiatry 19: 1017-1024.

Shabalin AA. 2012. Matrix eQTL: ultra fast eQTL analysis via large matrix operations. Bioinformatics 28: 1353-1358.

Smith GD, Ebrahim S. 2003. 'Mendelian randomization': can genetic epidemiology contribute to understanding environmental determinants of disease? Int J Epidemiol 32: 1-22.

Stegle O, Parts L, Piipari M, Winn J, Durbin R. 2012. Using probabilistic estimation of expression residuals (PEER) to obtain increased power and interpretability of gene expression analyses. Nat Protoc 7: 500-507.

Storey JD, Tibshirani R. 2003. Statistical significance for genomewide studies. Proc Natl Acad Sci 100: 9440-9445.

Stranger BE, Montgomery SB, Dimas AS, Parts L, Stegle O, Ingle CE, Sekowska M, Smith GD, Evans D, Gutierrez-Arcelus M, et al. 2012. Patterns of cis regulatory variation in diverse human populations. PLoS Genet 8: 272-284.

Sun L, Craiu RV, Paterson AD, Bull SB. 2006. Stratified false discovery control for large-scale hypothesis testing with application to genomewide association studies. Genet Epidemiol 30: 519-530.

Torres JM, Gamazon ER, Parra EJ, Below JE, Valladares-Salgado A, Wacher N, Cruz M, Hanis CL, Cox NJ. 2014. Cross-tissue and tissue-specific eQTLs: partitioning the heritability of a complex trait. Am J Hum Genet 95: 521-534.

Trapnell C, Pachter L, Salzberg SL. 2009. TopHat: discovering splice junctions with RNA-Seq. Bioinformatics 25: 1105-1111.

Veyrieras JB, Kudaravalli S, Kim SY, Dermitzakis ET, Gilad Y, Stephens M, Pritchard JK. 2008. High-resolution mapping of expression-QTLs yields insight into human gene regulation. PLoS Genet 4: e1000214.

Wang JB, Gamazon ER, Pierce BL, Stranger BE, Im HK, Gibbons RD, Cox NJ, Nicolae DL, Chen LS. 2016. Imputing gene expression in uncollected tissues within and beyond GTEx. Am J Hum Genet 98: 697-708.

Westra HJ, Peters MJ, Esko T, Yaghootkar H, Schurmann C, Kettunen J, Christiansen MW, Fairfax BP, Schramm K, Powell JE, et al. 2013. Systematic identification of trans eQTLs as putative drivers of known disease associations. Nat Genet 45: 1238-1243.

Zaas AK, Chen MH, Varkey J, Veldman T, Hero AO, Lucas J, Huang YS, Tumer R, Gilbert A, Lambkin-Williams R, et al. 2009. Gene expression signatures diagnose influenza and other symptomatic respiratory viral infections in humans. Cell Host Microbe 6: 207-217.

Received September 30, 2016; accepted in revised form May 1, 2017. 


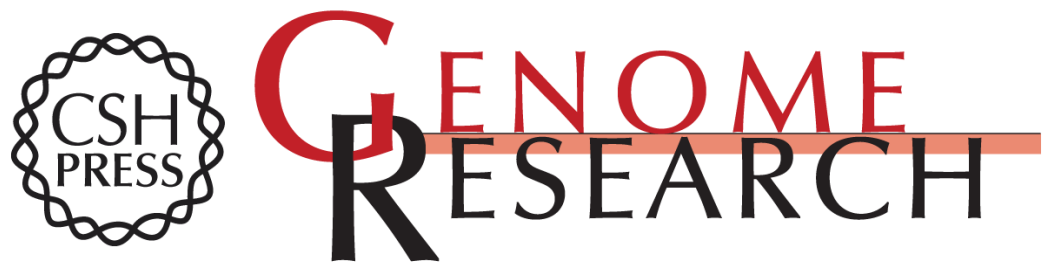

\section{Identifying cis-mediators for trans-eQTLs across many human tissues using genomic mediation analysis}

Fan Yang, Jiebiao Wang, The GTEx Consortium, et al.

Genome Res. 2017 27: 1859-1871 originally published online October 11, 2017

Access the most recent version at doi:10.1101/gr.216754.116

\section{Supplemental http://genome.cshlp.org/content/suppl/2017/10/04/gr.216754.116.DC1 \\ Material}

Related Content A genome-wide interactome of DNA-associated proteins in the human liver Ryne C. Ramaker, Daniel Savic, Andrew A. Hardigan, et al. Genome Res. November , 2017 27: 1950-1960 Co-expression networks reveal the tissue-specific regulation of transcription and splicing

Ashis Saha, Yungil Kim, Ariel D.H. Gewirtz, et al.

Genome Res. November , 2017 27: 1843-1858 Quantifying the regulatory effect

size of cis-acting genetic variation using allelic fold change

Pejman Mohammadi, Stephane E. Castel, Andrew A. Brown, et al.

Genome Res. November, 2017 27: 1872-1884

References This article cites 38 articles, 5 of which can be accessed free at:

http://genome.cshlp.org/content/27/11/1859.full.html\#ref-list-1

Articles cited in:

http://genome.cshlp.org/content/27/11/1859.full.htmI\#related-urls

Creative This article is distributed exclusively by Cold Spring Harbor Laboratory Press for the

License http://genome.cshlp.org/site/misc/terms.xhtml). After six months, it is available under a Creative Commons License (Attribution-NonCommercial 4.0 International), as described at http://creativecommons.org/licenses/by-nc/4.0/.

Email Alerting Receive free email alerts when new articles cite this article - sign up in the box at the Service top right corner of the article or click here.

\section{Affordable, Accurate Sequencing.}

